\title{
Effect of intermittent urethral catheterization and oxybutynin bladder instillation on urinary continence status and quality of life in a selected group of spinal cord injury patients with neuropathic bladder dysfunction
}

\author{
S Vaidyananthan, BM Soni, E Brown, P Sett, KR Krishnan J Bingley and S Markey \\ Regional Spinal Injuries Centre, District General Hospital, Town Lane, Southport, Merseyside, PR8 6PN, UK
}

\begin{abstract}
Objectives: A comparative assessment of (i) urinary continence status, (ii) quality of life, and (iii) sexuality in spinal cord injury patients prior to, and during intermittent catheterization with adjunctive intravesical oxybutynin therapy (Cystin: manufactured by Leiras Oy, Helsinki, Finland).

Setting: A hospital, and community-based study of selected adult, male, spinal cord injury patients registered with the Regional Spinal Injuries Centre, Southport.

Patients: Seven patients (mean age: 44.3 years) suffering from neuropathic bladder due to suprasacral spinal cord lesion of traumatic aetiology, and well settled in the community in the north-west of England were the subjects of this study. Before commencing the intermittent catheterization regime, these patients were on penile sheath drainage.

Intervention: Intermittent urethral catheterization was performed with sterile, single-use Nelaton catheters 5-6 times a day with intravesical instillation of oxybutynin $5 \mathrm{mg}$ in $30 \mathrm{ml}$, $1-3$ times a day for periods ranging from 14 to 30 months.

Outcome measures: Assessment of urinary continence, sexuality, and quality of life was made (i) at the outset before any intervention, (ii) during intermittent catheterization regime, and (iii) when the patients were using the oxybutynin bladder instillation along with intermittent catheterization.

Results: Initially all the seven patients were constantly wearing penile sheaths and leg bags. When these patients performed intermittent catheterization $5-6$ times in $24 \mathrm{~h}$, they attempted to discard the penile sheath during the day but they were experiencing mild to moderate urine leak between catheterization. They were compelled to wear penile sheaths during night. Subsequently, five patients took oxybutynin by mouth, but developed an unacceptable degree of side-effects necessitating discontinuation of the medication. Following commencement of intravesical oxybutynin therapy, all of them were able to discard the penile sheaths and leg bags during the day as well as during the night. However, on waking-up after a full night's sleep, three patients found dampness of their undergarments $1-2$ times per week. None of the patients experienced side-effects attributable either to the intermittent catheterization procedure, or to the intravesical oxybutynin therapy. The number of episodes of urinary infection requiring antibiotic therapy was $0.08 /$ patient $/$ month. All the seven patients noticed a remarkable improvement in the quality of life because they had achieved a high degree of continence. All the seven patients commented on the improved sense of their own sexuality which was attributed to (i) absence of incontinence episodes, (ii) improved self-image, and (iii) not wearing penile sheaths and leg bags.

Conclusion: These seven spinal cord injury patients achieved socially acceptable continence with improved quality of life, and enhanced sexuality with the intermittent urethral catheterization regime and intravesical oxybutynin therapy.
\end{abstract}

Keywords: Neuropathic bladder; intermittent catheterization; spinal cord injury; oxybutynin

\section{Introduction}

As a result of neuropathic dysfunction of the urinary bladder in patients with suprasacral spinal lesions of

Correspondence: S Vaidyanathan traumatic aetiology, the bladder no longer functions effectively as an organ for storage of urine. Further, these patients are not able to achieve complete emptying of their bladder at low intravesical pressures. Therefore, certain therapeutic measures need to 
be instituted to achieve satisfactory function of the lower urinary tract. In this context, many treatment options are available such as indwelling urethral catheter drainage, suprapubic catheter drainage, penile sheath drainage, abdominal urinary stoma, augmentation cystoplasty ${ }^{1}$, radio-frequency stimulation of sacral interior nerve roots with surgically implanted electrodes and receiver block, or intravesical capsaicin therapy $^{2}$. Preferably, the treatment option for a given patient is chosen by him/her after informed discussion with the health professionals. Of course, the final choice will depend on his/her personal life style, dexterity of his/her hands, care package that is available for the individual spinal cord injury patient, and the status of the upper and lower urinary tracts. All these variables are not static, and are likely to change from time to time. Therefore, the ideal treatment will be the one which is least invasive, and which does not cause any permanent alteration in his/ her body system. Thus the perfect treatment of the neuropathic dysfunction of the urinary bladder should be such that it helps the patient to simulate as closely as possible the life-style of an able-bodied individual, and does not produce any irrevocable change in his/her constitution. Therefore, surgical measures such as abdominal urinary stoma which may be of either the classical type, eg ureterostomy or ileal conduit urinary diversion requiring continuous wearing of an external urinary collecting device, or the newer operative procedures which offer urinary continence, and do not require the patient to wear a collecting device over the stoma, do not qualify as the treatment of choice in the initial stages after the spinal cord injury. These major interventions are reserved exclusively for those patients who could not manage their bladder satisfactorily with the less invasive treatment options, or for those individuals in whom these procedures are indicated in order to preserve the function of the kidneys.

As mentioned earlier, the first choice of treatment of the neuropathic dysfunction of the urinary bladder will be the one which helps the patient to adopt a life-style which is as close as possible to that of his pre-injury status. This means that he or she will not wear a catheter or a penile sheath, and will not wear a leg bag for continuous collection of urine. He/she will aim to achieve a socially acceptable degree of urinary continence, and the treatment regime will not impose restrictions on his/her social interactions. Ideally, any therapeutic intervention so chosen should not interfere with his/her sexuality and body image in that context. One young male paraplegic patient mentioned 'the penis should be readily available in an aesthetically acceptable condition for interaction with my partner as and when required'. From the medical and nursing perspectives, the chosen treatment regime should preserve the function of kidneys, and should neither produce any complication on its own nor should it make the individual more susceptible to other disease processes, eg he/she should not become more prone to getting urinary infection, or bladder neoplasm.

Within the above framework, it is believed that intermittent urethral catheterization does not alter the body image and helps to achieve complete, lowpressure emptying of the urinary bladder. In those patients in whom abnormally high pressures are generated within the bladder because of overactive detrusor function, oxybutynin hydrochloride will help to suppress the overactive detrusor function to some extent. Oxybutynin will help the patient to retain a larger amount of urine in the bladder, and this will be achieved at low intravesical pressures so that there is no detrimental effect upon the kidneys. However, oxybutynin when taken by mouth, may produce unwanted side-effects, the most predominant side-effect being dry mouth. With a dry mouth, the patient is tempted to drink more fluids, but by increasing his/her fluid intake, he/she will produce more urine which means either more frequent intermittent catheterization or urinary leakage between catheterization. Both these outcomes are not socially acceptable. To some extent, the sideeffect of dry mouth can be offset by spraying an artificial saliva preparation that is commercially available into his/her mouth and throat as often as it is required. The other option will be to administer the drug oxybutynin in to the urinary bladder directly so that the chances of experiencing the systemic side-effect of dry mouth are minimised. Intravesical administration of oxybutynin is well tolerated, devoid of side effects in the majority of the patients, provides subjective improvement in terms of continence, and is effective in patients who were refractory to regimens of oral medication with maximally tolerated doses of oxybutynin or could not tolerate any doses of oxybutynin because of the side effects ${ }^{3-5}$.

We studied the effect of intermittent catheterization and intravesical oxybutynin therapy on (i) the urinary continence status, (ii) quality of life, (iii) sexuality, and (iv) occurrence of side effects in selected male spinal cord injury patients with suprasacral lesions and neuropathic dysfunction of the urinary bladder. These patients had carried out the above therapeutic measures continuously at least for 12 months. As these variables are influenced to a great degree by the sex of the individual, only male patients were included in this investigation. It would be inappropriate to compare these patients performing intermittent catheterization and using oxybutynin bladder instillation on the status of urinary continence, quality of life, and sexuality, with those spinal cord injury patients who have gained continence either because they have recovered a great degree of neurological function, or they have undergone a surgical procedure such as implanted radio frequency stimulation of sacral anterior nerve roots, or augmentation cystoplasty. Therefore, in this study, each patient acted as his own control, and comparisons were made between the pre-treatment and post-treatment scores for each of the variables viz. continence, sexuality, and quality of life. 


\section{Patients and methods}

Seven patients suffering from neuropathic bladder due to suprasacral spinal cord lesions of traumatic aetiology, and well settled in the community in the north-west of England were the subjects of this study. The clinical details of these subjects are provided in Table 1. Prior to starting the intermittent catheterization regime, they were on penile sheath drainage. Intermittent urethral catheterization was performed with sterile, single-use Nelaton catheters 5-6 times a day. While performing intermittent catheterization, all the seven patients were experiencing mild to moderate degree of urine leak between catheterizations. Therefore, five patients took oxybutynin by mouth, but developed an unacceptable degree of side effects of oral oxybutynin necessitating discontinuation of medication. The remaining two patients, having learnt the possible side effects of oral oxybutynin therapy from their friends, did not want to take oxybutynin by mouth; they expressed their preference to try intravesical oxybutynin therapy straight away.

All the seven patients were then started on intravesical oxybutynin therapy (Cystrin: manufactured by Leiras Oy, Helsinki, Finland) consisting of bladder instillation of oxybutynin $5 \mathrm{mg}$ in $30 \mathrm{ml}, 1-3$ times a day which they have been using for periods ranging from 14 to 30 months. The following assessments were made (i) at the outset before any intervention, (ii) during the intermittent catheterization regime, and (iii) when the patients were using the oxybutynin bladder instillation along with intermittent catheterization.
Assessment of urinary continence

A spinal cord injury patient performing intermittent cathetherisation 5-6 times a day was defined as having achieved urinary continence if he did not wet his undergarments and was able to discard the penile sheath and the leg bag. He may be compelled to wear a penile sheath or a catheter when he undertakes (1) a long journey by air when there is no provision to perform urethral catheterization, (2) a whole day outing, or (3) a long evening out with friends for a 'drink'. The patient's overall satisfaction of his continence status was recorded on a scale ranging from zero (wet with urine most of the times, a predicament compelling him to wear a penile sheath and leg bag continuously for 7 days a week) to 100 (when he was dry most of the times and hence, he was able to discard the penile sheath and leg bag altogether).

\section{Assessment of sexuality}

The sexuality was defined from the patient's perspective on (i) sense of being attractive, (ii) ability and confidence in performing sexual acts without any embarrassing episode of urine leak, and (iii) body image as an attractive male sexual partner and his selfesteem. The question to be answered was whether this regime of intermittent catheterization and intravesical oxybutynin therapy enabled him to socialise without the need to wear a penile sheath or a catheter and a leg bag, and whether it facilitated him to perform his role as a male sexual partner in a more satisfying manner, or whether he experienced any embarrassing episode of urine leak while performing sex. The patient's overall

Table 1 Clinical details of the patients who were using intemittent catheterization and bladder instillation of oxybutynin solution and their scores on (i) urinary continence status, (ii) sexuality, and (iii) quality of life before any specific treatment was instituted for neuropathic bladder (Before), while they were performing intermittent catheterization (During IC), and while they were performing intermittent catheterization and also using bladder instillation of oxybutynin (IC + OXY)

\begin{tabular}{|c|c|c|c|c|c|c|c|}
\hline Patient identification & JR & KP & IB & RG & $\mathrm{DD}$ & TW & $\mathrm{BP}$ \\
\hline Year of birth & 1953 & 1957 & 1976 & 1929 & 1961 & 1938 & 1948 \\
\hline Year of injury & 1960 & 1975 & 1992 & 1988 & 1981 & 1986 & 1969 \\
\hline Level of injury & $\mathrm{T}-5$ & C-6 & $\mathrm{T}-9$ & $\mathrm{~T}-12$ & $\mathrm{~T}-5$ & $\mathrm{~T}-6$ & T-6 \\
\hline \multicolumn{8}{|l|}{ Continence score } \\
\hline Before & 0 & 0 & 0 & 0 & 0 & 0 & 0 \\
\hline During IC & 50 & 50 & 25 & 40 & 40 & 30 & 50 \\
\hline During IC + OXY & 99 & 95 & 95 & 95 & 95 & 95 & 95 \\
\hline \multicolumn{8}{|l|}{ Sexuality score } \\
\hline Before & 70 & 40 & 20 & 50 & 50 & 25 & 65 \\
\hline During IC & 80 & 60 & 20 & 65 & 70 & 50 & 80 \\
\hline During IC + OXY & 95 & 95 & 98 & 85 & 98 & 85 & 95 \\
\hline \multicolumn{8}{|l|}{ Quality of life score } \\
\hline Before & 85 & 50 & 30 & 50 & 50 & 30 & 60 \\
\hline During IC & 80 & 50 & 20 & 75 & 30 & 75 & 85 \\
\hline During IC + OXY & 99 & 85 & 99 & 95 & 95 & 99 & 95 \\
\hline \multicolumn{8}{|c|}{$\begin{array}{l}\text { Number of episodes of urinary infection } \\
\text { requring treatment with antibiotics } \\
\text { during the past twelve months: }\end{array}$} \\
\hline Before & 4 & 4 & 1 & 4 & 4 & 0 & 1 \\
\hline During IC + OXY & 1 & 3 & 0 & 0 & 1 & 1 & 1 \\
\hline
\end{tabular}


satisfaction about his sexuality while he adopted the intermittent catheterization regime with adjunctive intravesical oxybutynin therapy was noted on a scale of 0 (very unsatisfactory) to 100 (remarkably gratifying). The confidentiality of an individual's account of the status of his satisfaction in performance of sex was preserved.

\section{Assessment of quality of life}

The quality of life was given positive weightage if he was able to maintain urinary continence to an extent which was socially acceptable and he could (1) go on holidays, (2) socialise with his friends, (3) go for shopping, (4) visit his friend's homes, (5) receive guests in his home, or (6) pursue his studies or a vocation without inhibitions caused by urinary incontinence. This treatment plan of intermittent catheterization and intravesical oxybutynin therapy would have made a negative impact on the quality of life if it imposed unacceptable restrictions in his daily activities including his social life, or in the daily routine of his partner. The quality of life was judged by the patient on a scale of 0 (extremely miserable) to 100 (highly pleased when he was able to pursue all the aforesaid activities with confidence of being 'dry' after having discarded the penile sheath and the leg bag).

\section{Assessment of side-effects}

The occurrence of side effects such as urinary infection, or a stinging sensation after instillation of oxybutynin inside the urinary bladder was noted. The average number of episodes of urinary infection per patient per month was then calculated.

\section{Results}

None of the seven patients developed side effects of oxybutynin therapy such as dry mouth, visual disturbances or stinging sensation in the bladder following intravesical instillation of oxybutynin. This was in contrast to the status when five of these seven patients who took oxybutynin by mouth developed an unacceptable degree of side-effects necessitating discontinuation of the medication. Prior to any intervention strategy, all the seven patients were wearing penile sheaths and leg bags for $24 \mathrm{~h}$ a day. While performing intermittent catheterization only, they attempted to discard the penile sheath during the day but they experienced a mild to moderate degree of urine leak between catheterization. However, during the night, they were compelled to use penile sheaths and leg bags. With the addition of oxybutynin bladder instillation to the intermittent catheterization regime, all the seven subjects were able to discard the penile sheath and leg bag during the day as well as during the night. However, on waking-up after a full night's sleep, three patients found dampness of their undergarments 1-2 times per week. Their scores on continence, sexuality, and quality of life improved during the treatment regime with intermittent catheterization and bladder instillation of oxybutynin (Table 1). All the seven patients commented on the improved sense of their own sexuality which was attributed to (i) absence of incontinence episodes, (ii) improved self-image, and (iii) not wearing penile sheaths and leg bags. Four patients, who had a stable partner, experienced a profound improvement in the quality of their sex life. Confidence in their continence status allowed freedom to wear shorts, and to go swimming without the attachment of penile sheath and leg bag. This ability to discard the penile sheath and absence of embarrassing episodes of urine leak was the most remarkable achievement of this therapeutic regimen. Other advantages of this treatment modality as perceived by these patients were: (i) reduction in washing of personal clothes and bed sheets because they achieved a high degree of urinary continence; (ii) feeling of well being; and (iii) greatly improved quality of life. Their comments included: 'I would be very upset to go back to wearing penile sheaths'; 'I feel fantastic-over the moon!'; 'This has revolutionised my life'; 'Totally changed my body image'; 'A lot better; I feel elated'; 'I am pleased; I am much more confident'; ' $100 \%$ better; I feel almost normal'; 'I can wear shorts; I can go for swimming without feeling embarrassed about the leg bag or leaking urine'. Of course, all of them noted that they should be able to locate a wheel chair accessible facility for performing intermittent catheterization when they went out for more than $6 \mathrm{~h}$ at a stretch.

\section{Discussion}

This study shows that oxybutynin bladder instillation as an adjunct to an intermittent catheterization regime could help selected spinal cord injury patients with a suprasacral type of neuropathic bladder to achieve a satisfactory degree of urinary continence and discard the use of penile sheath and leg bag. However, this treatment is expensive as the cost of oxybutynin bladder instillation (Cystrin, Pharmacia \& Upjohn Ltd, Davy Avenue, Knowhill, Milton Keynes MK5 8PH, UK) is $£ 33.00$ plus VAT for ten vials of $5 \mathrm{mg}$ in $30 \mathrm{ml}$. The other brand of oxybutynin (Ditropan) bladder instillation is not available at present (personal communication from Sally Cox, Senior Medical Information Officer, Lorex Synthelabo Ltd, Globe Park, Marlow, Bucks SL7 1LW, UK). Therefore, stringent selection criteria have been developed by us for intravesical oxybutynin therapy (see Appendix) and we now follow this protocol when choosing a patient for this treatment regime.

During an episode of acute urinary infection, these patients may experience episodes of urine leak; however, with treatment of urinary infection by oral antibiotics, the embarrassing episodes of urine leak stop. Another common cause of urine leak in a patient who was doing well on this regime is the development of a bladder stone. Following removal of bladder 
stone(s), eg by electrohydraulic lithotripsy, we have seen patients regaining their continence on the intermittent catheterization and adjunctive oxybutynin bladder instillation regime. Intravesical oxybutynin therapy may not abolish completely the overactive detrusor function in some patients and one plausible explanation might be the status of the vesical mucosal innervation ${ }^{6}$. Therefore, it should be remembered that oxybutynin bladder instillation is not a panacea for urinary incontinence in spinal cord injury patients; it is a useful addition to the available treatment regimes for the management of the neuropathic bladder with low compliance. Perhaps, when tolterodine (a new muscarinic receptor antagonist, which has selectivity for bladder smooth muscle over salivary glands ${ }^{7}$, and which is administered by mouth), is granted a product licence, the indications for intravesical oxybutynin therapy might become limited even further.

\section{References}

1 Mast $\mathrm{P}$, et al. Experience with augmentation cystoplasty. A review. Paraplegia 1995; 33: 560-564.

2 Cruz F, et al. Desensitization of bladder sensory fibres by intravesical capsaicin has long lasting clinical and urodynamic effects in patients with hyperactive or hypersensitive bladder dysfunction. Journal of Urology 1997; 157: 585 - 589.

3 Vaidyanathan S, Soni BM, Fraser MH, Krishnan KR. Oxybutynin in spinal cord injured patients with neuropathic bladder. Reviews in Contemporary Pharmacotherapy 1994; 5: $217-223$.

4 Madersbacher H, Jilig G. Control of detrusor hyperreflexia by the intravesical instillation of oxybutynin hydrochloride. Paraplegia 1991; 29: $84-90$.

5 Krishnan KR, et al. A double-blind, randomised, placebocontrolled, parallel-group, multi-center study of intravesical oxybutynin in the symptomatic relief of urge incontinence in patients with detrusor instability/hyperreflexia. Neurourology and Urodynamics 1996; 15: 674-676.

6 Vaidyanathan S, et al. Nerve fibres in urothelium and submucosa of neuropathic urinary bladder: an immunohistochemical study with S-100 and neurofilament. Paraplegia 1996: 34: 137-151.

7 Abrams $\mathrm{P}$, et al. A randomised, double-blind, placebocontrolled, dose-ranging study of the safety and efficacy of tolterodine in patients with detrusor hyperreflexia. Abstracts of the International Continence Society 26th Annual Meeting, 27-30 August 1996. International Continence Society, Athens, Greece. pp $276-277$.

\section{Appendix}

Flow chart for selection of patients with neuropathic bladder due to suprasacral spinal cord lesions of traumatic aetiology for the treatment regime of intermittent catheterization and intravesical obybutynin therapy

1 Adult spinal cord injury patients with neuropathic dysfunction of the urinary bladder due to suprasacral spinal cord lesions of traumatic aetiology who are in the age group of 18 to 75 years, well settled in the community and registered with a Regional Spinal Injuries Centre, and in whom intermittent urethral catheterization is indicated on medical grounds may be considered for this treatment regime. Patients who had undergone surgical procedure(s) upon the lower urinary tract to facilitate bladder emptying, eg bladder neck resection, and/or division of external urethral sphincter should not be considered for participation in this therapeutic regimen, as these operative procedures are likely to make them incontinent and dribble urine. The overall treatment plan is then discussed with the patient and if he agrees to it we proceed further.

2 Intermittent urethral catheterization: The patient is given a booklet on intermittent catheterization. A video on the technique of urethral catheterization is shown to the patient, his partner and the carers when applicable. Initially, a doctor performs the urethral catheterization and makes an assessment as to whether the patient can perform catheterization safely. If for any medical reason, eg urethral sphincter spasm, or the presence of urethral stricture, the procedure of self-catheterization is considered unsuitable for a given patient, an alternative therapeutic measure is considered for that patient. Subsequently, the patient or the carer performs the urethral catheterization under medical/nursing supervision till such a time he/she is able to do so satisfactorily, and with confidence. If the patient is unable to continue with intermittent urethral catheterization for any reason, or is unwilling to continue with the intermittent catheterization schedule, he is at liberty to change to an alternative treatment plan, eg indwelling urethral catheter drainage. If the patient does not leak urine while performing intermittent catheterization 5-6 times a day, he continues to perform regular intermittent catheterization, and he does not require the adjunctive oxybutynin therapy.

3 On the other hand, if a spinal cord injury patient with a suprasacral lesion, despite performing intermittent catheterization 5-6 times a day has been experiencing urine leak, and urodynamics revealed overactive detrusor function, low bladder compliance, he is prescribed oxybutynin tablet by mouth provided there is no contraindication to the administration of oxybutynin. The starting dose of oxybutynin is $2.5 \mathrm{mg} 2-3$ times a day and the dose of oxybutynin is increased in a stepwise manner to a maximum dose of $5 \mathrm{mg} 4$ times a day, if required. The dose of oxybutynin is increased if the spinal cord injury patient experienced significant urinary leakage between catheterization on more than two occasions in a week, and he had not developed any major side effect of the oral oxybutynin therapy.

4 If the patient is happy with the degree of urinary continence he had achieved with the oral oxybutynin therapy together with the intermittent catheterization regime, he continues with that treatment strategy, and he is not considered for the intravesical oxybutynin therapy. 
5 Indications for intravesical oxybutynin treatment The majority of spinal cord patients will achieve continence either with intermittent catheterization, or with a combination of intermittent catheterization and oral oxybutynin treatment. However, there are two groups of patients amongst those performing intermittent catheterization in whom the intravesical route of administration of oxybutynin is indicated. The first category includes those patients who are unable to take oxybutynin by mouth because of unacceptable side effects. The second category includes a few patients who continue to have significant urine leaks despite performing intermittent catheterization 5-6 times a day, and taking the maximum dose of oxybutynin by mouth, viz. $5 \mathrm{mg} 4$ times a day. For those select group of patients who have been unsuccessful with oral oxybutynin therapy either because of unacceptable side effects, or because of lack of efficacy of oxybutynin when it is administered by mouth, the intravesical route of administration of oxybutynin should be considered.

6 The technique of intravesical administration of oxybutynin is demonstrated to the patient, his partner, and carers when applicable. Subsequently, the patient or his carer performs the task of administration of intravesical preparation of oxybutynin into the urinary bladder through the catheter inserted per urethra after emptying the bladder of its contents of urine. In case for any reason the patient or his carer is unable to achieve proficiency in this task, or they are unwilling to pursue with it, or in the rare event of his developing a major side effect to the intravesical oxybutynin therapy, an alternate therapeutic modality is considered for that patient, eg indwelling urethral catheter drainage, suprapubic cystostomy, etc.

7 The patient or his carer performs the task of intravesical administration of $5 \mathrm{mg}$ of oxybutynin 3 times a day for a week. If the patient achieved reasonable degree of urinary continence, he continues with the regime of instilling $5 \mathrm{mg}$ of oxybutynin into his urinary bladder 3 times a day. If however, he has significant urinary leakage between urethral catheterization, the timing of catheterization and administration of oxybutynin will require reviewing. Even after making such adjustments on the time schedules for catheterization and for oxybutynin bladder instillation, if he continues to experience urine leaks, the frequency of intravesical administration of $5 \mathrm{mg}$ of oxybutynin is then increased from 3-4 times a day.

8 Informed discussion is held by the health professionals with the patient, his partner and carers about the additional input required in the care package to implement the therapeutic intervention of intermittent catheterization and intravesical oxybutynin therapy. If the spinal cord injury patient is happy to continue with intermittent catheterization and intravesical oxybutynin therapy, this treatment protocol is then discussed with his General Practitioner and the District Nurse and their cooperation is solicited in carrying out this treatment plan. It would be appropriate to consult the Regional Pharmaceutical Adviser as well, at this stage. The officials of the Health Authority of the region where the patient resides are then contacted and the financial implication of this treatment plan is discussed. Intravesical oxybutynin at the moment falls within the category of an unlicensed medication which can only be used 'on a named patient' basis. It is, therefore vital that prior approval is obtained from the appropriate Regional Health Authority of the National Health Service for the funding of the intravesical oxybutynin therapy in the community, as the cost of treatment has to be accommodated within the budget of that particular Health Authority.

9 If for any reason, particularly because of changed social circumstances, it is considered inappropriate to continue with this therapeutic regime of intermittent catheterization and intravesical oxybutynin therapy, necessary modifications are made in the treatment plan as the patient's overall wellbeing and happiness is always the prime concern in planning any treatment plan.

10 If after 2 weeks of trial with intravesical oxybutynin therapy, the patient continues to develop significant urine leak despite (i) performing intermittent catheterization 5-6 times a day, (ii) restricting the total fluid intake to 1.5 litres a day, (iii) restricting the number of cups of tea/ coffee to a reasonable number, and (iv) instilling oxybutynin $5 \mathrm{mg}$ intravesically 4 times a day, he is considered for an alternative treatment plan, eg indwelling urethral catheter drainage.

11 While the patient is practising this treatment regime of intermittent catheterization and intravesical oxybutynin therapy, he is reviewed once in 48 weeks by the medical personnel at a Regional Spinal Injuries Centre. 